\title{
Age-related decline of sleep-dependent consolidation
}

\author{
Rebecca M.C. Spencer, ${ }^{1}$ Arvin M. Gouw, and Richard B. Ivry \\ Department of Psychology and Helen Wills Neuroscience Institute, University of California, Berkeley, California 94720-1650, USA
}

\begin{abstract}
Sleep-dependent memory consolidation is observed following motor skill learning: Performance improvements are greater over a $12-\mathrm{h}$ period containing sleep relative to an equivalent interval without sleep. Here we examined whether older adults exhibit sleep-dependent consolidation on a sequence learning task. Participants were trained on one of two sequence learning tasks. Performance was assessed after a 12-h break that included sleep and after a 12-h break that did not include sleep. Older and younger adults showed similar degrees of initial learning. However, performance of the older adults did not improve following sleep, providing evidence that sleep-dependent consolidation is diminished with age.
\end{abstract}

A diverse set of literature indicates that sleep facilitates memory consolidation. Performance on perceptual (Karni et al. 1994) and motor skill (Walker and Stickgold 2004) tasks improves following sleep. For example, in producing learned sequences of finger movements, performance improves more after a 12-h interval with sleep than after a 12-h interval awake. Since initial performance does not differ between training in the morning or evening (Walker et al. 2002; Spencer et al. 2006), the improvement is unlikely to be due to variations in circadian cycles or arousal. Rather, these performance changes are hypothesized to reflect sleep-dependent consolidation (SDC) of learning (Walker and Stickgold 2004).

It has been proposed that SDC may decrease with age, due to changes in sleep rhythms (Hornung et al. 2005), cortisol levels (Buckley and Schatzberg 2005), or other factors. However, the effect of age on SDC has not been directly examined. In the present study, we tested older adults on explicit and implicitcontextual versions of a sequence learning task. Previously, we demonstrated SDC on the performance of these tasks in young adults (Spencer et al. 2006) whereas SDC was not observed on an implicit noncontextual sequence learning task. This dissociation was in accord with the hypothesis that SDC may be restricted to tasks that engage the hippocampus. We now compare the performance of older participants to that of the younger participants, evaluating age-related effects on off-line consolidation of learning.

We tested 32 right-handed individuals (17 males, 15 females) ranging in age from 45 to $80 \mathrm{yr}($ mean $=59.0 ; \mathrm{SD}=11.1)$. The sample of younger adults reported in Spencer et al. (2006) was composed of 38 younger adults (17 males, 21 females, mean $=20.8 ; \mathrm{SD}=2.1$ ). Although all of the younger participants were tested prior to the older participants, the testing location, apparatus, and procedure were the same for both groups. We excluded participants who had been diagnosed with sleep disorders or were taking medications known to affect sleep. Procedures were approved by the institutional review board at UC Berkeley and informed consent was provided by all participants.

Half of the participants in each age group were assigned to the "explicit" group and half to the "implicit-contextual" group. Both tasks were modified versions of the serial reaction time task (Nissen and Bullemer 1987). Manual responses were made with the left hand to stimuli presented on a computer monitor. Four horizontally aligned white boxes were displayed at all times. An " $\mathrm{X}$ " appeared in one of the boxes and remained on the screen

\section{'Corresponding author.}

E-mail rspencer@berkeley.edu; fax (510) 642-5293.

Article is online at http://www.learnmem.org/cgi/doi/10.1101//m.569407. until a response was made (Fig. 1A,B). The next stimulus appeared $300 \mathrm{msec}$ later, initiating the next trial. Trials were grouped into blocks of 70 trials. Instructions emphasized speed. Feedback indicating mean reaction time (RT) and total errors was provided at the end of each block.

On sequence blocks, the stimuli followed a 10-element sequence that cycled repeatedly. For the explicit group, this was a sequence of locations, and participants responded by pressing the key in the corresponding spatial position on a response board (Fig. 1A). Participants were informed that the stimuli would follow a fixed sequence on most blocks and that they should learn the sequence so that they could respond as fast as possible.

For the implicit group, responses were based on the color of the $X$ (Fig. 1B). The color of the $X$ followed a 10-element color sequence and the location of the $\mathrm{X}$ followed a different 10element sequence. Thus, while responding on the basis of location would be inaccurate, the location information provided a contextual cue - the sequence of stimulus colors was presented in the context of the sequence of stimulus locations (see Mayr 1996; Helmuth et al. 2000). Participants were not informed of the presence of either sequence. We selected this task because learning on this task is facilitated by sleep (Spencer et al. 2006).

Prior to the experiment, participants in the implicit group were taught the mapping between the four colors and their associated responses (e.g., pinky for yellow; index for red). The experimenter described the color mapping. Then sample stimuli were presented and the participant pressed the appropriate keys. The experimenter reminded the participant of the mapping if necessary and continued practicing until it was evident that the participant had learned the color mapping. During the experiment, a $1.5-\mathrm{cm}$ diameter sticker above each response key indicated the color assignment for that key. Participants were told that this was to serve as a reminder between blocks and that they should not look at them on a trial-by-trial basis. The experimenter monitored the participants to make sure there was minimal use of the stickers during the blocks.

For both tasks, learning was assessed in each session by the inclusion of random blocks. For the explicit group, the location of each stimulus was selected at random (Fig. 1C). For the implicit group, the stimulus color and location were independently selected at random (Fig. 1D). The randomization procedure was constrained to match key features of the sequence blocks. Two of the four locations appeared twice and two items appeared three times in each 10-item sequence in the sequence blocks. Thus, random blocks maintained the same proportional presentation of each stimulus location (Fig. 1C,D). In addition, stimulus repetitions (e.g., 1-1 or 3-3) were not allowed nor were three-element trills (such as 1-3-1 or 3-2-3). Repetitions and trills may enhance 
A

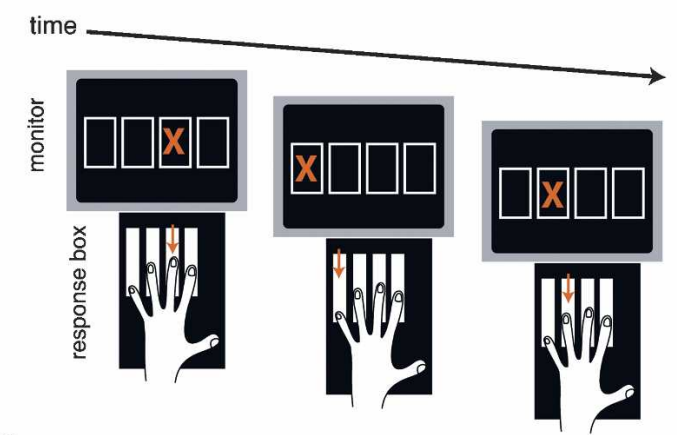

C

Sequence block: 4-2-1-4-3-1-2-4-1-3... 4-2-1-4-3-1-2-4-1-3... Random block: $3-1-4-2-1-4-3-2-4-1 \ldots$ 4-1-3-4-1-3-2-4-1-2...
B

Implicit Contextual Sequence Learning Task

time

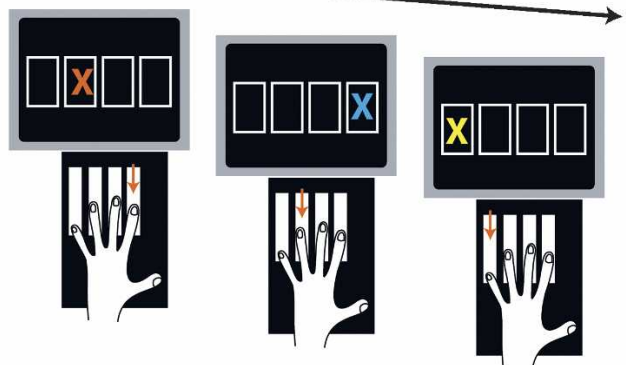

D

$\begin{array}{lll}\text { Sequence block: } & -2-1-4-3-1-2-4-1-3 \ldots & -2-1-4-3-1-2-4-1-3 \ldots \\ \text { Random block: } & 3-1-4-2-1-4-3-2-1-1 . & 4-1-3-1-1-3-2--1-2 \ldots \\ \text { Phase-shift block: } 2-1-4-3-1-2-4-1-3-4 \ldots & 2-1-4-3-1-2-4-1-3-4 \ldots\end{array}$

Figure 1. (A) In the explicit sequence learning task, participants pressed the key (arrows) corresponding to the position of a visual stimulus, an X. (B) In the implicit contextual task, responses were based on the color of the X. Example of stimuli for the explicit (C) and implicit contextual $(D)$ tasks. The color indicates the color of the $\mathrm{X}$ and the number indicates the location of the $\mathrm{X}$.

sequence awareness and exaggerate learning measures (Vaquero et al. 2006).

Sequence learning was operationalized as the difference in mean RT on the random blocks compared to the mean RT for the preceding and following sequential blocks. To measure contextual learning in the implicit condition, we included a phase shift block in which the location sequence was shifted with respect to the color sequence. In this manner, the color (and response) sequence remained unchanged (Fig. 1D). Increases in RT on the phase shift blocks compared to the surrounding sequence blocks reflect disruption of contextual associations between the colors and locations.

Participants were tested in three sessions. For the explicit condition, session one contained 11 blocks. Stimuli were randomized in blocks 8 and 10. Given the addition of a phase shift block in the implicit-contextual condition, 14 blocks were included in session one. The colors and locations were randomly selected in blocks 7 and 13; block 10 was a phase shift block. For both groups, sessions two and three contained seven blocks. The stimuli were randomly selected in block 6 . For the implicitcontextual group, block 4 served as a phase shift probe.

To assess off-line changes in performance, sessions were separated by a break that either included or did not include sleep. The order of the wake and sleep intervals was counterbalanced. Half the participants in each group (AM-PM-AM group) began session 1 between 7 and 9 a.m. and half of the participants (PMAM-PM group) began session 1 between 7 and 9 p.m. Sessions 2 and 3 began 12 and 24 h, respectively, after session 1 . For example, participants in the AM-PM-AM group might be trained at 8 a.m. (session 1) and learning would be assessed at 8 p.m. that day (session 2) and again at 8 a.m. the following day (session 3). A signature of SDC in sequence learning tasks is that performance is better immediately after a break that includes sleep compared to a break that does not include sleep (e.g., Walker et al. 2002, 2003).

We note that the current design does not include control conditions to evaluate generic changes in performance across sessions (e.g., recovery from fatigue). Such controls have been included in many previous studies of SDC (Walker et al. 2002; Mednick et al. 2003; Spencer et al. 2006). For example, Spencer et al. (2006) included a control condition for the explicit task, training a group of participants with stimulus locations that were selected at random on all blocks. There was no reliable decrease in RT following sleep; thus, the between-session improvements observed following sleep in the explicit group were specific to sequence learning. Given the difficulty in recruiting older participants for a three-session study, we chose to not repeat this control here.

The participant's perceived alertness was assessed at the end of each session on a scale of 1 ("drowsy") to 7 ("wide awake"). Another questionnaire was administered at the end of the third session to determine when sleep occurred during the overnight break and whether or not the participant had taken a nap during the daytime break. Additionally, participants in both groups were surveyed at the end of session 3 to determine whether they thought the stimuli appeared sequentially or at random. They were then informed that there had been a sequence and were asked to generate a 10-element sequence.

Participants had little difficulty performing the tasks. Overall, the mean percentage of errors for the older adults (explicit: $3.1 \%$; implicit: $4.2 \%$ ) was similar to that of younger adults (explicit: 2.8\%; implicit: 3.1\%). Accuracy did not differ across blocks (explicit: $F_{(24,399)}<0.1$; implicit: $F_{(27,420)}=1.1, P=0.33$ ) or sessions ( $F<1$ for both conditions). As expected, RTs were considerably slower for the implicit task due to the arbitrary mapping between the colors and response keys, as well as the absence of awareness. When compared to performance of young adults (Spencer et al. 2006), the mean RTs were considerably slower for the older adults (explicit: $413 \mathrm{msec}$ vs. $203 \mathrm{msec}$; implicit: 653 msec vs. 456 msec).

In each session older adults slowed on the random block, indicating that they had learned the sequence. This cost was evident on both tasks (Fig. 2A). A two-way ANOVA compared block type (random probe block and the average of the surrounding sequence blocks) and session. The main effect of block type was significant (explicit: $F_{(1,90)}=26.7, P<0.001$; implicit: $\left.F_{(1,90)}=10.4, P=0.002\right)$. The main effect of session was not significant (explicit: $F_{(2,90)}=1.8, P=0.17$; implicit: $F_{(2,90)}=2.2$, $P=0.11$ ) nor was the interaction ( $F<1$ for both groups), suggesting that performance for the older participants was relatively stable after session 1. Older adults also showed evidence of contextual learning on the implicit task; RTs were slower when the phase relationship between stimulus color and location was altered, disrupting contextual associations $\left(t_{(15)}=3.4, P=0.002\right)$.

We used two methods of analysis to compare sequence learning for the two age groups. The first analysis was based on 
A

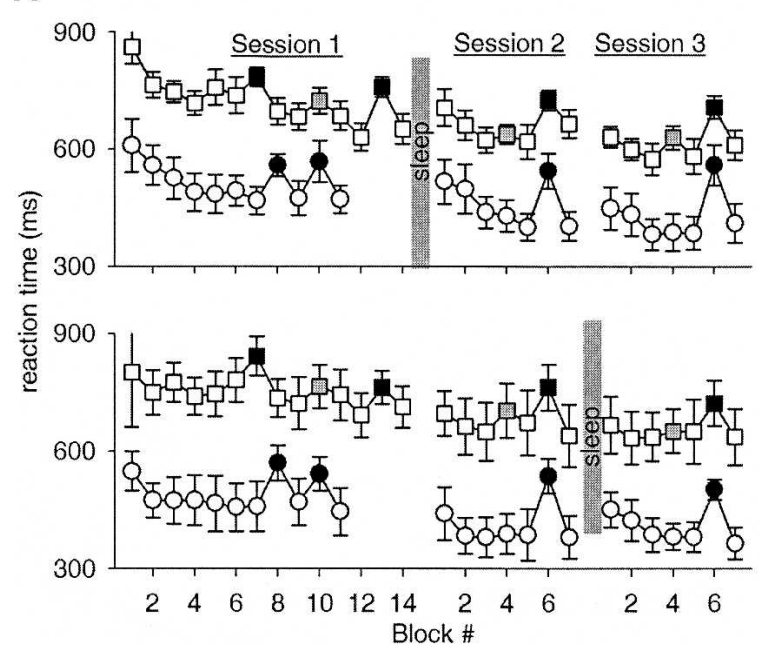

B

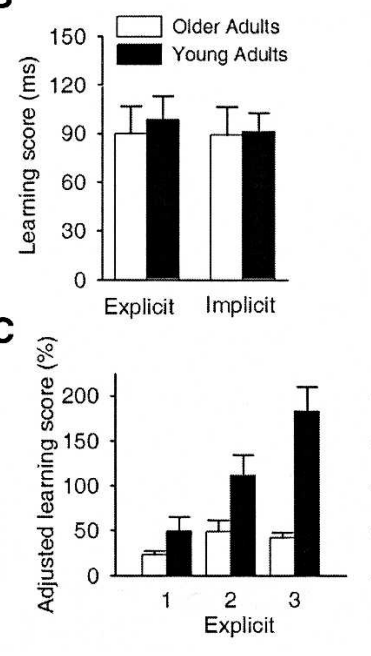

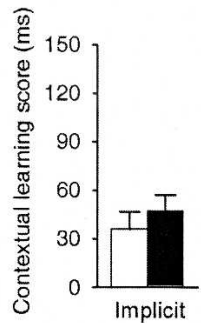

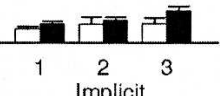

Figure 2. $(A)$ Learning curves for the older adults. The explicit (circles) and implicit-contextual (squares) sequence learning tasks were performed in three sessions each separated by $12 \mathrm{~h}$. Half of the participants performed the first session in the evening (PM-AM-PM; top panel) and half performed the first session in the morning (AM-PM-AM; bottom panel). Within each session, sequence learning was assessed by comparison of random blocks (black symbols) to surrounding sequence blocks (white symbols). For the implicit task, contextual learning was probed by disrupting the between-dimension sequence of response-relevant colors and locations (gray symbols). (B) Increase in RT on random and phase shift blocks (implicit task only) during session 1 collapsed across the two session orders. (C) Adjusted learning scores (see text) for each session.

the absolute increase in RT on the probe blocks. Based on the random probes, the magnitude of learning did not differ between the two age groups (Fig. $2 \mathrm{~B}$; explicit: $F_{(1,90)}=1.2, P=0.25$; implicit: $\left.F_{(1108)}=3.3, P=0.08\right)$ nor did age interact with session ( $F<1$ for both groups). Similarly, the increase in RT on the phase shift probes (RT on the phase shift probe minus the RT for the surrounding sequence blocks) was similar for the two age groups and across sessions, nor was the interaction term of these two factors significant (Fig. 2B; all $F<1$ ).

The above analyses do not take into account the difference in mean RT for the age groups. Given this difference in overall speed, we used a normalized measure in the second set of analyses, dividing the increase on each probe block by the mean RT of the surrounding sequence blocks; in essence, this measure looks at learning in terms of a proportional increase in RT on the probe blocks. When calculated this way, the effect of age was significant for the explicit task $\left(F_{(1,90)}=31.1, P<0.001\right)$ as was the age $\times$ session interaction $\left(F_{(2,90)}=6.0, P=0.003\right)$. The latter was due to the fact that the adjusted learning score increased significantly across sessions for the young participants, but not the older participants (Fig. 2C). For the implicit task, neither of the main effects nor the age $\times$ session interaction were significant (all $F<1$ ). The phase shift probe of contextual learning also failed to show an aging effect $\left(F_{(1108)}=1.3, P=0.24\right)$.

To evaluate whether performance of older adults was more affected by the time of the day, we looked at the interaction of age $\times$ time of day of session 1 (morning for AM-PM-AM group; evening for PM-AM-PM group). The interaction was not significant (Fig. 3; explicit and implicit, all $F<1$ ). Thus, while the older participants were considerably slower overall, this effect was present to a similar degree in the morning and evening sessions.

Off-line consolidation was measured by subtracting the mean RT for the first two blocks in session $n$ from the last two sequence blocks in session $n-1$. These values were normalized by dividing by the mean RT on the first two blocks in session $n$. This "difference value" has been used in previous studies of SDC (Walker et al. 2002, 2003; Spencer et al. 2006) and is especially useful here since it adjusts for the substantial age effect on RT. Based on the hypothesized deficit in SDC with age, of particular interest were improvements in performance for intervals separated by sleep.

The older adults failed to show any indication of improvement on either task between sessions, a null effect observed in both the explicit $\left(t_{(15)}=-1.06, P=0.86\right)$ and implicit conditions $\left(t_{(15)}=-1.09, P=0.85\right)$. Indeed, on both tasks, the mean RT on the first two blocks after sleep was slower than the mean RT on the last two blocks prior to sleep (Fig. 3). When a direct comparison was made between the two age groups, younger adults showed significantly greater reductions in RT after 12 -h breaks with sleep on both tasks (explicit: $F_{(1,28)}=33.2, P<0.001$; implicit: $\left.F_{(1,34)}=15.9, P<0.001\right)$. These results are consistent with the hypothesis that SDC decreases with age (Buckley and Schatzberg 2005; Hornung et al. 2005). The same pattern of results was obtained when the analyses were repeated using absolute RT difference.

We also looked at changes in performance over a 12-h break without sleep. In our previous study (Spencer et al. 2006), younger adults showed a significant decrease in RT over this interval on the explicit task and not the implicit-contextual learning task. While the older adults also showed a decrease in RT (5 msec) over this interval in the explicit condition, this effect was not reliable overall (difference values relative to zero $t_{(15)}=1.2$, $P=0.12$ ). However, the group of older adults who began testing in the morning (AM-PM-AM) did show a significant reduction in RT across this interval $\left(t_{(7)}=3.1, P<0.001\right)$. The older adults showed a reliable decrease in RT on the implicit task after a 12-h break without sleep (mean $\left.=25 \mathrm{msec}, t_{(15)}=1.8, P=0.05\right)$. When we compared the two age groups, there was a significant age $\times$ task interaction $\left(F_{(1124)}=20.7, P<0.001\right)$. We do not have an account of this crossover interaction, especially since the younger adults did not show a reduction in RT on the implicit task immediately after a 12 -h break without sleep.

Could the lack of SDC in the older adults be due to changes in sleep patterns that occur with age? While polysomnography measures were not obtained, sleep diaries were maintained. The older adults averaged $7.0 \mathrm{~h}(\mathrm{SD}=0.9)$ of sleep. This value does not differ from that of the younger adults (mean $=7.3 \mathrm{~h}$; $\mathrm{SD}=1.2$ ). Thus, the lack of off-line learning in the older adults 


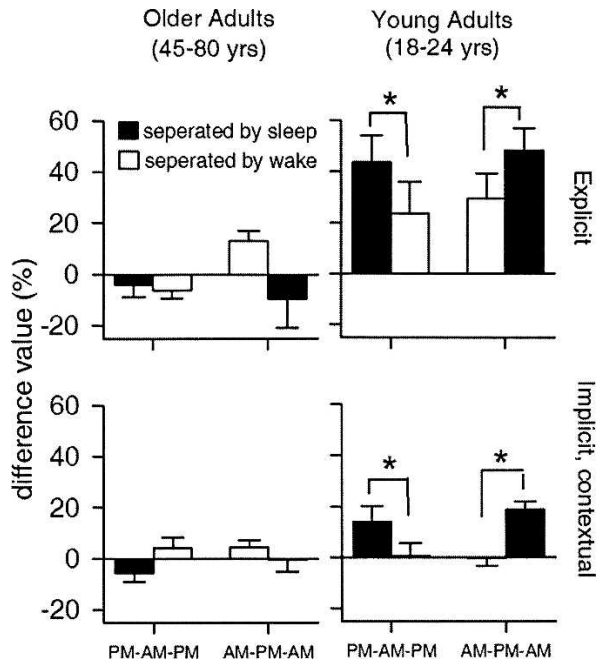

Figure 3. Off-line changes in performance following 12-h break with sleep (black) or without sleep (white).

was not associated with a decrease in subjective sleep duration. We also considered the possibility that the lack of a difference between the sleep and wake intervals might be due to napping, given that a nap alone can lead to SDC (Mednick et al. 2003). If the older participants took naps in the AM-PM interval, the null effects might be because SDC occurred during both breaks. Two aspects of the results argue against this hypothesis. First, the mean RTs after each break were not different than at the end of the preceding session. Second, only six participants (four implicit; two explicit) reported taking a nap. Analysis of the data after excluding these participants did not change the results.

It is also unlikely that the observed effects reflect differences in circadian rhythms for the age groups. As reported above, the interaction between age and time of day was not significant for the initial learning scores (Fig. 2B). Moreover, the participants' perceived alertness did not differ across sessions $\left(F_{(1,62)}=3.3\right.$, $P=0.10$ ) or age groups (main effect and interactions: $F<1$ ). On the 7-point scale, the mean perceived alertness was 6.1 for the younger adults and 5.8 for the older adults across sessions. Alertness did not correlate with SDC (young, $r=0.21$; older, $r=-0.12)$ as measured by a sleep benefit score calculated as the difference of the difference values (i.e., [AM-PM] - [PM-AM]). In addition, this sleep benefit score was neither related to the amount of time participants were awake after the first (or only) evening test session (young, $r=0.09$; older, $r=0.11$ ) nor to the amount of time awake prior to the post-learning morning test session (young, $r=-0.19$; older, $r=-0.04$ ). Thus, while we do not have any direct measures of circadian rhythms, individual differences in sleep-wake cycles were not predictive of SDC.

Taken together, these results reveal an age effect on measures of off-line memory consolidation. Despite relatively normal initial learning, the older participants failed to demonstrate evidence of memory consolidation in conditions in which consolidation is observed in younger participants. The absence of a change in performance was most evident when the 12-h break included sleep supporting the hypothesis that SDC decreases with age.

Although research on the neural correlates of SDC is in its infancy, we propose three nonmutually exclusive reasons why this learning mechanism may be diminished with age. First, older adults might have a general problem with off-line consolidation mechanisms. The relationship between sleep-dependent and sleep-independent consolidation mechanisms is a subject of considerable interest. A recent study points to distinct off-line consolidation processes for intervals that include or do not include sleep (Cohen et al. 2005). Our results on this issue are ambiguous. While the results are mixed regarding age-related changes in sleep-independent consolidation, we did find a marked impairment in sleep-dependent consolidation. It is unclear if this pattern reflects a dual impairment in memory consolidation processes or if the sleep-dependent component reflects an amplification of an impairment in off-line consolidation. This is an important subject for future research.

Second, SDC may decrease due to changes in sleep quantity or quality (Danker-Hopfe et al. 2005). While the overall quantity of sleep did not differ for younger and older adults, time spent in the relevant sleep stage may differ. The degree of SDC for explicit skill learning correlates with time spent in NREM-2 sleep (Walker et al. 2002). However, the amount of NREM-2 sleep does not decrease with age and, in fact, may increase (Danker-Hopfe et al. 2005). Rather, changes in the qualities of the sleep stages, including NREM-2, may occur with age, even if the duration is unchanged (Gaudreau et al. 2001). For instance, the number of sleep spindles may decrease with age (for review, see De Gennaro and Ferrara 2003). Sleep spindles have been hypothesized to promote synaptic plasticity during sleep, increasing following learning and correlating with SDC (Fogel and Smith 2006; Nishida and Walker 2007).

Finally, aging may effect the functional operation of neural systems associated with off-line learning. We have proposed that SDC, at least for the motor skill tasks used here, is dependent on the hippocampus (Spencer et al. 2006). Indeed, the implicitcontextual task was chosen because of its hypothesized dependency on hippocampal learning mechanisms (Chun and Phelps 1999; Keele et al. 2003; Cohen et al. 2005). SDC is not observed for noncontextual implicit learning, learning which is independent of the hippocampus. Age-related changes in hippocampal function (Sapolsky 1992; Buckley and Schatzberg 2005) may underlie the absence of SDC in older adults. Direct measurements of physiological markers associated with sleep or the functionality of neural systems involved in learning should prove useful in elucidating the mechanisms of the age-related decline in SDC.

\section{Acknowledgments}

R.M.C.S. was supported by NIH grant F32 NS048012.

\section{References}

Buckley, T.M. and Schatzberg, A.F. 2005. Aging and the role of the HPA axis and rhythm in sleep and memory-consolidation. Am. J. Geriatr. Psychiatry 13: 344-352.

Chun, M.M. and Phelps, E.A. 1999. Memory deficits for implicit contextual information in amnesic subjects with hippocampal damage. Nat. Neurosci. 2: 844-847.

Cohen, D.A., Pascual-Leone, A., Press, D.Z., and Robertson, E.M. 2005. Off-line learning of motor skill memory: A double-dissociation of goal and movement. Proc. Natl. Acad. Sci. 102: 18237-18241.

Danker-Hopfe, H., Schäfer, M., Dorn, H., Anderer, P., Saletu, B., Gruber, G., Zeitlhofer, J., Kunz, D., Barbanoj, M.-J., Himanen, S.L., et al. 2005. Percentile reference charts for selected sleep parameters for 20to 80-year-old healthy subjects from the SIESTA database. Somnologie 9: 3-14.

De Gennaro, L. and Ferrara, M. 2003. Sleep spindles: An overview. Sleep Med. Rev. 7: 423-440.

Fogel, S.M. and Smith, C.T. 2006. Learning-dependent changes in sleep spindles and Stage 2 sleep. J. Sleep Res. 15: 250-255.

Gaudreau, H., Carrier, J., and Montplaisir, J. 2001. Age-related modifications of NREM sleep EEG: From childhood to middle age. $J$. Sleep Res. 10: $165-172$.

Helmuth, L.L., Mayr, U., and Daum, I. 2000. Sequence learning in Parkinson's disease: A comparison of spatial-attention and number-response sequences. Neuropsychologia 38: 1443-1451.

Hornung, O.P., Danker-Hopfe, H., and Heuser, I. 2005. Age-related changes in sleep and memory: Commonalities and 
interrelationships. Exp. Gerontol. 40: 279-285.

Karni, A., Tanne, D., Rubenstein, B.S., Askenasy, J.J.M., and Sagi, D. 1994. Dependence on REM sleep of overnight improvement of a perceptual skill. Science 265: 679-682.

Keele, S.W., Ivry, R.B., Mayr, U., Hazeltine, E., and Heuer, H. 2003. The cognitive and neural architecture of sequence representation. Psychol. Rev. 110: 316-339.

Mayr, U. 1996. Spatial attention and implicit sequence learning: Evidence for independent learning of spatial and nonspatial sequences. I. Exp. Psychol. Learn. Mem. Cogn. 22: 350-364.

Mednick, S., Nakayama, K., and Stickgold, R. 2003. Sleep-dependent learning: A nap is as good as a night. Nat. Neurosci. 6: 697-698.

Nishida, M. and Walker, M.P. 2007. Daytime naps, motor memory consolidation and regionally specific sleep spindles. PLOS ONE 2: e341. doi: 10.1371/journal.pone.0000341.

Nissen, M.J. and Bullemer, P. 1987. Attentional requirements of learning: Evidence from performance measures. Cognit. Psychol. 19: $1-32$.

Sapolsky, R.M. 1992. Stress, the aging brain, and the mechanisms of neuron death. MIT Press, Cambridge.

Spencer, R.M.C., Sumn, M., and Ivry, R.B. 2006. Sleep-dependent consolidation of contextual learning. Curr. Biol. 16: 1001-1005.

Vaquero, J.M.M., Jimenez, L., and Lupianez, J. 2006. The problem of reversals in assessing implicit sequence learning with serial reaction time tasks. Exp. Brain Res. 175: 97-109.

Walker, M.P. and Stickgold, R. 2004. Sleep-dependent learning and memory consolidation. Neuron 44: 121-133.

Walker, M.P., Brakefield, T., Morgan, A., Hobson, J.A., and Stickgold, R. 2002. Practice with sleep makes perfect: Sleep-dependent motor skill learning. Neuron 35: 205-211.

Walker, M.P., Brakefield, T., Seidman, J., Morgan, A., Hobson, J.A., and Stickgold, R. 2003. Sleep and the time course of motor skill learning. Learn. Mem. 10: 275-284.

Received March 6, 2007; accepted in revised form May 25, 2007. 


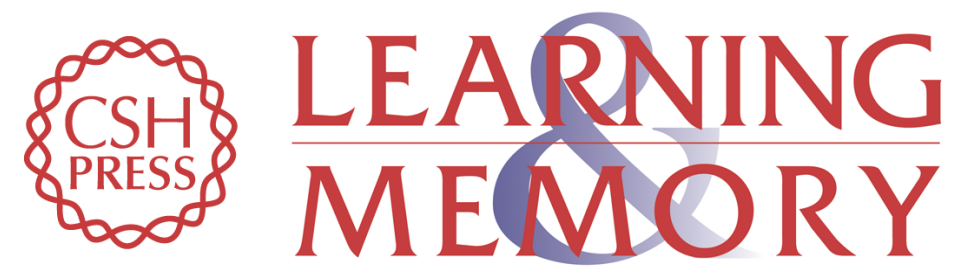

\section{Age-related decline of sleep-dependent consolidation}

Rebecca M.C. Spencer, Arvin M. Gouw and Richard B. Ivry

Learn. Mem. 2007, 14:

Access the most recent version at doi:10.1101/lm.569407

References This article cites 20 articles, 3 of which can be accessed free at: http://learnmem.cshlp.org/content/14/7/480.full.html\#ref-list-1

License

Email Alerting Receive free email alerts when new articles cite this article - sign up in the box at the Service top right corner of the article or click here. 